Proceedings of the 2015 Winter Simulation Conference

L. Yilmaz, W. K. V. Chan, I. Moon, T. M. K. Roeder, C. Macal, and M. D. Rossetti, eds.

\title{
USING SIMULATION TO ASSIST RECRUITMENT IN SEASONALLY DEPENDANT CONTACT CENTERS
}

\author{
Leeanne May \\ School of Computer Science \\ University of Nottingham \\ Jubilee Campus \\ Nottingham, NG8 1BB, UK
}

\author{
Peer-Olaf Siebers \\ School of Computer Science \\ University of Nottingham \\ Jubilee Campus \\ Nottingham, NG8 1BB, UK
}

\begin{abstract}
The weather is unpredictable and can have a large impact on the profitability of seasonal businesses, particularly if staffing requirements are highly temperature-dependent. In this paper we describe our efforts in developing a what-if analysis tool to assist affected Small and Medium Enterprises in determining the best case scenario for timing hiring new staff and deciding the optimum length of temporary employment contracts. Together with a boiler maintenance company we have developed a prototype simulation tool that can be employed by users with minimal statistical and modelling knowledge. Our usability tests with the boiler maintenance company confirmed the usefulness of the developed tool as a decision support aid for managers. In this paper we focus on describing the tool development and testing process. With regards to real world experimentation we are still waiting for the feedback from the company.
\end{abstract}

\section{INTRODUCTION}

Payroll costs to companies can make up anywhere from 18-50\% of operating expenses (SHRM 2008). For businesses which must substantially increase their full time equivalent (FTE) members of staff during peak periods, it is important to get the timing of recruitment correct to avoid unnecessary costs. When the peak period is decided by something as variable as the weather, this decision is particularly difficult.

There has been a lot of work carried out in the area of Contact Centers (CC) and hospitals to determine the optimum number of staff needed based on the service requirements of the business (e.g. Ernst et al. 2004). Less research has been conducted into what period of time is needed to achieve the staffing levels required. For example, it may take over a month to recruit and train a new employee, and over seven weeks before they are fully trained (CFA 2012). If recruitment starts too early then staff will have increased idle time; recruit too late and the work increases faster than staff can cope, leading to complaints and lost customers. Since some businesses hire up to three times as many FTE at peak times, this can cause significant damage to business if misjudged (Myron 2002).

Energy companies, for instance, are heavily affected by the weather. Temperature is the main driver of gas use in the UK (CelsiusPro 2015). This means that the changes in temperature can be a huge strain on boilers and thus boiler repair companies. Academic work to date, which includes ambient temperature measurement of "degree-days" as a way to forecast consumption (Quayle and Diaz 1980) has focused on energy companies. In our study we work with a company that provides boiler maintenance and repair services. As gas-based central heating systems are the primary way energy customers heat their homes, we can apply the same principles to a boiler repair and service company (Meier and Rehdanz 2010). 
Forecast models are analytical models that do not provide any information about the dynamics of the system over time (Leombruni and Richiardi 2005). When studying systems that are complex, dynamic, and stochastic in nature, simulation is generally recognized as one of the best support technologies, and a valuable aid in the strategic, tactical, and operational decision making process (Siebers and Aickelin 2008). A simulation model consists of a set of rules that define how a system changes over time, given its current state. It provides an insight into system dynamics rather than just predicting the output of a system based on specific inputs. However, combining both modelling approaches might provide even better insight into the operations of a system.

One of the biggest challenges our company's CC faces each year is to estimate the required CC's staffing levels over the winter period, when it cannot be known in advance how severe the winter will be. Our aim is to develop a novel simulation tool that helps managers to make better informed decisions about their CC recruitment needs. For this purpose we want to integrate the ideas of "degree-days" consumption forecasting into a stochastic simulation model. The tool is also intended to support managers to estimate effective lengths of contracts for temporary staff.

\section{BACKGROUND}

\subsection{Contact Centers}

Around 1 million people are employed in the CC industry in the UK (CCA Global 2015). A CC is the department (or group of departments) that handle customer enquiries. It can either be the customer-facing operations department of a company, or a company on its own to which others outsource work (CFA 2012). In contrast to traditional call centers, which only handle phone calls, CCs manages phone calls, emails, electronic chat and post, all of which have their own assumptions and data requirements. In this paper we focus on CCs but findings could equally be applied to traditional call centers.

A CC quality is judged by its ability to meet a set of Key Performance Indicators (KPIs). These alter slightly from company to company; however, they generally fall into the following categories: First call resolution; average handling time; after call work time; service level; abandonment rate. A CC manager's job is to attempt to balance these indicators by matching the number of staff required to peak call times. Getting this prediction wrong can lead to customer dissatisfaction and increased stress for employees; in addition, overstaff and the labor costs can be very high.

\subsection{Human Resource Processes}

Recruitment can be carried out by a Human Resources (HR) department, individual line managers or, most commonly in CC businesses, a mix of the two. The time taken for recruitment is the sum of several different activities: placement of an advert, assessment of applications, accepting candidates, and candidate training. The overall time these activities take will be in the range of several weeks. At any point a preferred applicant can drop out, wasting a lot of time and money. The risk of a potential staff member leaving is worse for CCs than other industries, with a $25 \%$ rate of average turnover versus 18 $20 \%$ for the rest of the economy (CWU Research 2004). As a result, predicting the number of CCs which will be required is a difficult task even if the number and timing of vacancies is known well in advance.

\subsection{Companies Affected by the Weather}

Almost every home in the UK has a central heating system that is powered by either gas or electricity, and all systems need maintenance. Colder weather forces customers to contact the company taking care of their property when something goes wrong with their heating systems. Most customers with boiler cover will also need to book a service each year. Therefore, historic call data is affected by a mix of service due times and the severity and timing of the winter months. In these scenarios the unknowable impact of the 
May and Siebers

severity of the weather can make planning the staff resources required very difficult, especially if the company does not have years of historical data to use as a baseline from the different types of winter.

\subsection{Measuring Weather Patterns Using Degree-Days}

Degree-days are a standard unit for determining the energy needed to heat a building, and can be used as an alternative to mean temperature when measuring weather patterns. They have been shown to have a correlation with heating costs to a $99 \%$ confidence level (Meier and Rehdanz 2010). To calculate them, one takes a relevant temperature for the scenario $\left(\right.$ e.g. $\left.15^{\circ} \mathrm{C}\right)$ and works out for how long and how many degrees the temperature is below that amount. For example, if the temperature of interest is $15^{\circ} \mathrm{C}, 24$ hours at $13^{\circ} \mathrm{C}$ would be two degree days. One benefit of degree-days is that the comparator number can be changed as needed; for example, it may be better to compare with $17^{\circ} \mathrm{C}$ depending on what the measurement is to be used for. Degree-days also increase with the cold, which is easier to follow when comparing it to incoming call rates and finally, the measure of degree-days has a stronger correlation with incoming calls than mean temperature.

\subsection{Operations Research Approaches to Contact Centre Staffing Requirement Analysis}

There are two common ways for CCs to predict staffing levels in house: spreadsheet models or software (Ernst et al. 2004). Spreadsheet models use historic data to predict staffing levels. The modeler uses assumptions such as the number of calls an advisor can take an hour and historic numbers of incoming calls to estimate the necessary number of staff. Spreadsheets can generate random numbers and some statistical distributions to improve the accuracy of the prediction. Spreadsheets are cheap, need a low level of skill to use effectively and are familiar to office workers; however, they are restricted in their ability to show the interaction of different elements, and can become very unwieldy in size and complexity (Robinson 2014). These spreadsheet models can be used to forecast short term staff needs, such as how many people are needed at different parts of the day or week, and also to suggest how many people will be needed at different parts of the year. CC staffing software is usually based on telephone traffic theory (a specialized version of queuing theory). An example implementation of this theory can be found in the Erlang-C traffic model (Koole and Mandelbaum 2002). Academics in the area of queuing theory argue that there are many limitations to this method. These include the assumptions of which distributions are used and that all customers and advisors are "statistically identical". True human behaviour of customers (e.g. patience level) and advisors (e.g. fatigue) is not taken into account within this approach (Brown et al. 2005). A week of planning can be achieved but long term effects on calls are more difficult to plan for.

Complex mathematical approaches have also been applied to solving CC staffing problems. Some approaches are discussed in Ernst et al. (2004). Constraint programming has been shown to be useful when the workspace is rigid but it can be slow and uncompromising. Set-covering approaches can be too heavily simplified to be effective. A multi-method approach, such as constraint processing combined with linear programming's branch and bound has been shown to get around some of these issues.

In effect all operational research mathematical approaches are effective for CC staffing but have some shortcomings. They rely on a very high level of abstraction and strong assumptions; they focus only on operations and do not take into account diversity in human behaviour. Another shared limitation is the reliance on historical data. As will be shown later, this is very risky.

\subsection{Simulation}

Simulation is one of the most powerful tools available to decision makers responsible for the design and operation of complex processes and systems. Robinson (2014) identifies three key advantages of simulation modelling in comparison to other modelling methods: (1) the variability inherent in the real world system can be modelled; (2) the number of assumptions required is dependent on the time and data 
available, not the methodology; (3) the results are transparent to the end user. But simulation is not without its disadvantages: (1) it is an expensive option; (2) it is time-consuming and can require a lot of data; (3) it can lead to overconfidence due to its apparent transparency. Therefore it is always important to consider the results of a simulation as part of a wider cost-benefit analysis (Robinson 2014).

There are three core simulation modelling paradigms: System Dynamics (SD), Discrete Event (DE), and Agent-Based (AB). These can also be combined in many ways to form hybrid models. SD modelling is process oriented. It was first developed to investigate economic and social systems like the adoption of a new product. The technique considers the physical system as a network of feedback loops with a high degree of mutual interconnection, making it useful for high level process modelling (Forrester 1997). This simulation modelling approach will not be suitable for our case as it requires a high level of aggregation. If we were interested in how different departments affect each other, this method would be more applicable. In contrast we are interested in the interaction of individuals within the system. DE modelling is process oriented. Each process begins with a source, which generates entities; they will then pass through queues and delays that represent the system (Robinson 2014). Typically the technique is used to model manufacturing processes or other processes that lend themselves well to queues. This emphasis on queuing also lends itself to $\mathrm{CCs}$, as different types of call can be created and split according to type, then enter a queue until an advisor is available to deal with the call. AB modelling is object oriented. Here a system is modelled as a collection of autonomous decision-making entities called agents. Each agent individually assesses its situation and makes decisions on the basis of a set of rules (Bonabeau 2002). AB models are essentially decentralized; there is no place where global system behaviour would be defined. Instead, the individual agents interact with each other and their environment to produce complex collective behaviour patterns. With regards to our CC scenario we could consider creating a heterogeneous population of customer and advisor agents and take some true human behaviour for customers (e.g. patience level) and advisors (e.g. fatigue) into account. But as a CC is process oriented it would make more sense to develop a hybrid model: a process based system (using a DE approach) which contains active objects (using agents) to get the best from both worlds.

\subsection{Call Centre and Contact Centre Simulation Examples}

We did not find many examples of CC simulation models. There are, however, many examples of call center simulation models. Here we present both types. We have picked two samples from the help section of the multi-paradigm simulation software AnyLogic (XJtek 2015) which we briefly describe here.

The example "Call Centre" is a DE simulation that allows the user to manually change call arrival rates and call service times. The main use of this model is that it allows the user to see the effect of practicing cross skilling, in which advisors are trained to handle different types of calls so that they can answer calls from other departments when queues get too long.

The example "Customer Support Centre" is a much more complex hybrid DE/AB simulation, where requests of different types and complexities are generated through a more realistic method that increases during peak periods of the day, with user-configurable peak levels. The staff who deal with the requests are modelled as state-based agents. New agents are generated each week as set by the user; these agents are removed over time from the available resource so that they can take part in training and improve in skill set and expertise. This model can be used to determine the effects of separating different types of tasks, such as answering emails or calls between different types of skilled advisors, and includes the effect of advisors leaving the system.

\section{TOOL DEVELOPMENT}

We aim to develop a novel tool to support $\mathrm{CC}$ decision making for staffing levels in response to winter pressures. For this purpose we use a case study approach. Our partner for this project is a boiler maintenance and repair company which is facing the problem described above every year. Staff of the 
May and Siebers

partner company have supported us throughout the tool development process, from the conceptualization through to the user testing. They also have provided data where there was none publically available.

\subsection{Conceptualization}

For the conceptualization of our simulation model we followed Robinson's conceptual modelling framework (Robinson 2014). We have organized several focus groups with different stakeholders from the partner company as well drawn information from the literature.

- Problem: A boiler maintenance and repair company receive calls from customers throughout the year to book appointments. Service calls stay reasonably constant throughout the year, however breakdown calls increase during colder months. Staffing levels need to be increased in order to meet service level targets, however level is determined by financial constraints. The company needs a simulation to assist managing the risk of hiring levels decided.

- Objectives: Consistently meet these KPIs from June to May: average time in system of customers $<6$ min.; abandonment rate $<5 \%$; calls answered within $20 \mathrm{sec}$. $>90 \%$; calls per advisor per hour: 6-8.

- Constraints: At least 3 member of CC staff must be on shift; CCs receive calls 24/7.

- Experimental factors (means by which it is proposed that the modelling objectives are to be achieved): FTE available at model initialization; target FTE; winter severity level; customer base number; abandonment rate distribution; contract length of new staff.

- Responses (measures used to identify whether the objectives have been achieved): Calls answered (number of calls that reach the sink when the call was not abandoned); calls abandoned (number of calls that reach the sink when the call was abandoned); answered less than 20 seconds (number of calls that where answered after queuing for less than 20 seconds); number of advisors available (total number of advisors available to work that day); time in system (length of time the call is in the call process system). Data was collected between $8 \mathrm{am}$ and $8 \mathrm{pm}$. This is the standard daytime operating hours of companies that offer boiler repair and servicing (British Gas 2015; nPower 2015).

- Required Data: Distribution of calls and staff throughout the day (Source: Public data about typical peak times was used); call data (Source: Company data including number of calls by queue, time waited in queue and outcome (abandoned or answered)); fixed term contract lengths (Source: Questionnaire); average time till advisor leaves (Source: Questionnaire).

- Assumptions: Weather and recruitment are not affected by location in the UK; one member of staff interacts with one customer at a time; customers who have abandoned calls do not call again.

- Simplifications: Customers calling in multiple times is included as part of arrival rate; two types of calls i.e. services and breakdowns are included, but not sales calls; staff members leave according to distribution, there is no difference between staff members who resign or are dismissed; customer base is static; recruitment process focus on training time; we consider unlimited queues - no dropped calls.

- Content: Calls are included as they are key inputs (type, outcome, time in queue, time in system); advisors are included as they are key inputs (type, time till leave, experience level); queues and service points are included as they are key processes (allow time out, service time); weather is included as it is a key input (degree-days); recruitment is included as it is a key input (time for training, time till experienced); time is included as it is a key variable (day, month).

- Agent Classes: We have decided to use a hybrid DE/AB approach. We model the CC as a queuing system but incoming calls and advisors as agents. For our first model we have followed the KISS principle (Axelrod 1997) and kept things simple. We use "passive" agents, which are basically individual objects with a list of attributes to give them a personal identity. Currently they do not initiate any actions and do not have any specific goals. Later these agent classes can be further developed into fully fledged "active" agents (as described in the conclusion section).

- Processes: We have two separate processes in our model which are represented in Figure 2 and 3 using logic flow diagrams. These are "call process" and "recruitment process". 
May and Siebers

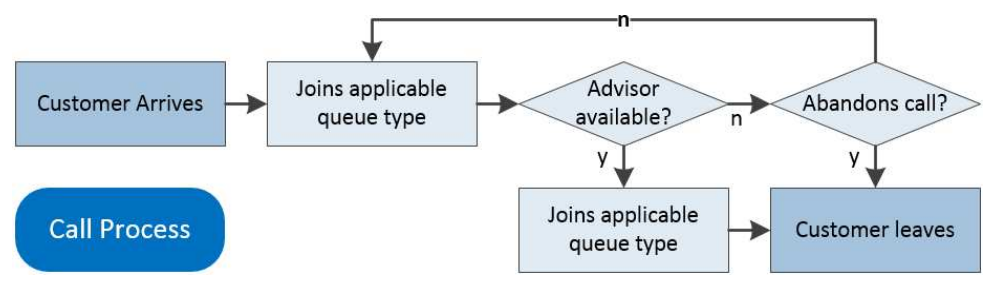

Figure 2: Call process.

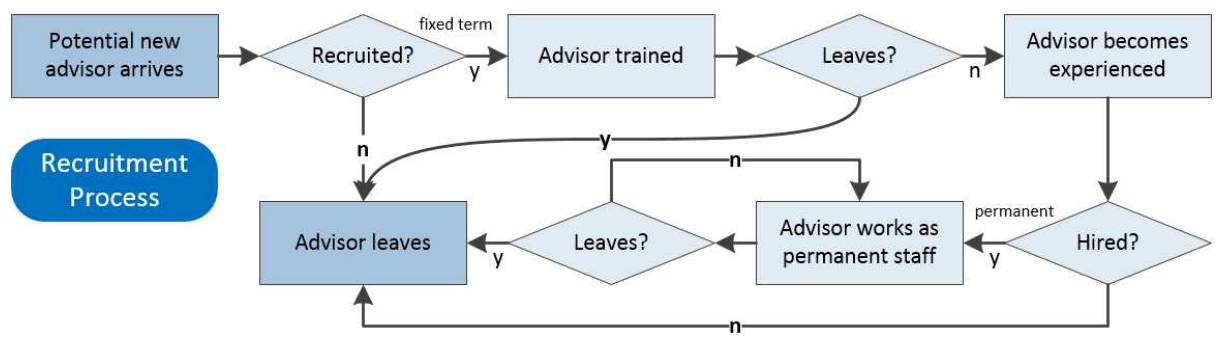

Figure 3: Recruitment process.

\subsection{Implementation}

The model described in Section 3.1 has been implemented AnyLogic 7.0 (University Edition) and extended by our own Graphical User Interface (GUI) to form a prototype tool. AnyLogic is a multiparadigm Eclipse-based commercial drag and drop modelling and simulation IDE. It can be programmed and extended using Java and supports GUI design through a range of template element like buttons and sliders. We have used an iterative approach while implementing the model. First we create a limited model that could take inputs of data from industry and provide realistic outputs. This was then adapted to accept changing variables required for experimentation. It was subsequently exported as a Java applet so that it is accessible without the need to install any software.

\subsubsection{Agent Classes}

In our simulation all customers are represented as calls. A Call Agent (CAgent) class was defined so that individual call objects could be created, each with their own set of attributes. The most important attribute is dblTimeTiliAbandon, which is assigned when the object is created. In CC simulations, time to abandon is traditionally either ignored or assumed to follow an exponential distribution. Using real-world data and distribution-fitting, we found that a lognormal distribution provides a much better fit as it follows the gradient more closely. Table 1 shows the results for the two different call types considered. The prefix "entity" provides access to the custom fields of that specific entity. Depending on the type of call (service or breakdown), a time to abandon is assigned as a random number from within the related distribution when the entity is created. The proportion of calls abandoned fitted within the expected range from the scenario and so this was determined to be reasonably valid. A full investigation into the effects of different distribution rates can be found in May (2014).

Table 1: Result of distribution-fitting for "time till abandon".

\begin{tabular}{|l|l|}
\hline \multicolumn{1}{|c|}{ Call Type } & \multicolumn{1}{|c|}{ Time Till Abandon } \\
\hline Service & entity.dblTimeTillAbandon = lognormal $(4.11051,1.36609,0) / 60 ;$ \\
\hline Breakdown & entity.dblTimeTillAbandon = lognormal $(4.56343,1.44683,0) / 60 ;$ \\
\hline
\end{tabular}


The CC Advisor Agent (CCAgent) class provides a template for CC advisors. These entities are affected by the process at numerous times (as shown in Figure 3). First, when created, they are assigned an expected time of leaving. For permanent agents this is a period of a few years to reflect benchmarking studies (CWU Research 2004) with some advisors leaving sooner to reflect people at different stages of their career. For fixed-term agents a period was chosen to reflect short-term contract length.

Three contract lengths were considered: 3, 6, and 9 months. One was chosen as it reflected the approach taken by the company and the other two after considering the patterns of historic winter weather. Triangular distributions were used to assign the random length to dblTimeTillLeave because the data was not available. The min, max and mode were chosen based on the reply to a questionnaire. Once initialized, an agent would be assigned a new value for dblTimeTillleave if their type changed from fixed-term to permanent. The other attribute that would change for a CCAgent during its lifetime is the level of experience. As the advisor passed through the stages of training and time passes, they become more effective. The effectiveness level is then applied to the service times to reflect the level of experience within the current set of staff. We based the values for these levels on information informally gathered during chats with managers. Details about distributions and levels are provided in Table 2.

Table 2: "Time till leave" distribution settings and "effectiveness" levels.

\begin{tabular}{|l|c|c|c|c|}
\hline \multirow{2}{*}{ CCAgent Object } & \multicolumn{3}{c|}{ Time till Leave } & \multirow{2}{*}{ Effectiveness } \\
\cline { 2 - 4 } & min & max & mode & \\
\hline Permanent & 4 weeks & 3 year & 1 years & 1 \\
\hline Fixed-Term Training & 2 weeks & 7 months & 6 months & 0.6 \\
\hline Fixed-Term Experienced & 2 weeks & 7 months & 6 months & 0.8 \\
\hline
\end{tabular}

\subsubsection{Processes}

For defining our processes we utilized the AnyLogic process modelling library. The key elements we used are "Source" (generates agents), "Service" (seizes resource units for the agent, delays it, and releases the seized units), "Sink" (disposes incoming agents), and "ResourcePool" (provides resource units that are seized and released by agents).

The model of the recruitment process after the implementation in AnyLogic is shown in Figure 4a. The simulation opens with a set of experienced CCAgents. The user sets this number and also the total number of staff they wish to have employed by mid-October that the simulation will attempt to match. At the start of September the simulation starts injecting a randomly determined amount of new starters each week to represent assessment centers. The advisor has a chance of passing the assessment center and begins one week of basic training. There is no time here because this effectively represents behaviour that begins in August with the placement of adverts. After the first week of training, the advisors receive phone training and at this point are counted as a resource - though not very productive. After training some agents leave and the rest spend three months becoming experienced. During this time their effectiveness improves until finally some are kept on permanently and others complete their fixed-term contract. The advisors were able to timeout whilst becoming experienced or long term employed to reflect people leaving the company. As stated before, $\mathrm{CC}$ staff turnover is 5\% higher than the national average and it was important to reflect that instability. The 6 and 9 month recruitment scenarios have a shorter phase to reflect the positive aspect of longer contract lengths that less time needs to be spent on recruitment. The 3 month contracts allow managers to be more flexible with the recruitment process, but needed a longer recruitment period to take into account the higher chance of staff turnover.

The model of the call process after the implementation in AnyLogic is shown in Figure 4b. The Recruitment Process feeds into the Call Process through the setting of the number of units available to the resource pool that supports the service blocks. Following the conceptual model, calls are sorted according 
to their type, breakdown or service, and are directed to the correct service block. They wait there until either an advisor is free or they reach their preset time-to-abandon, at which point they go to the sink where the call object is destroyed.

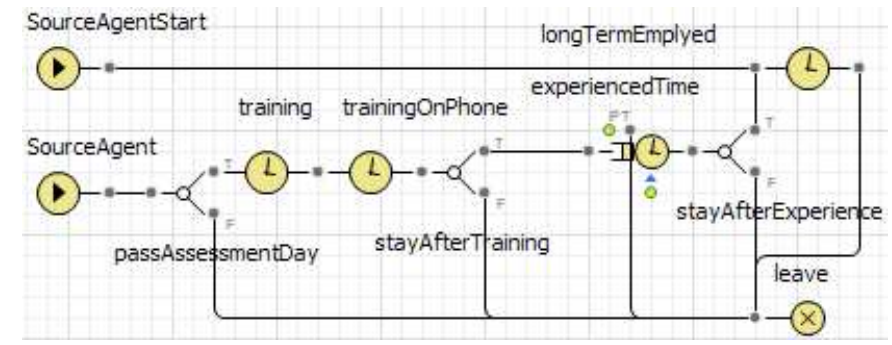

a. recruitment process model

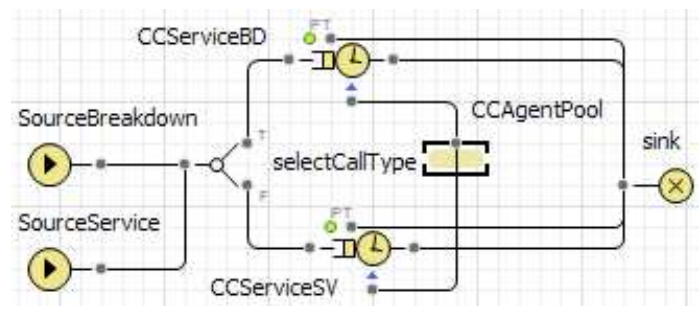

b. call process model

Figure 4: Graphical representation of processes modelled in AnyLogic.

\subsubsection{Key Model Elements}

Each of the two processes described in Section 3.2.2 has two source blocks to generate the different types of calls and advisors. Each source block produces entities at a specific rate and assigns unique values to the attributes of the created entities. The distribution of staff required to answer $90 \%$ of calls within 20 seconds and of call arrivals throughout a typical day was defined by using data from a company that offered rostering support (Figure 5).
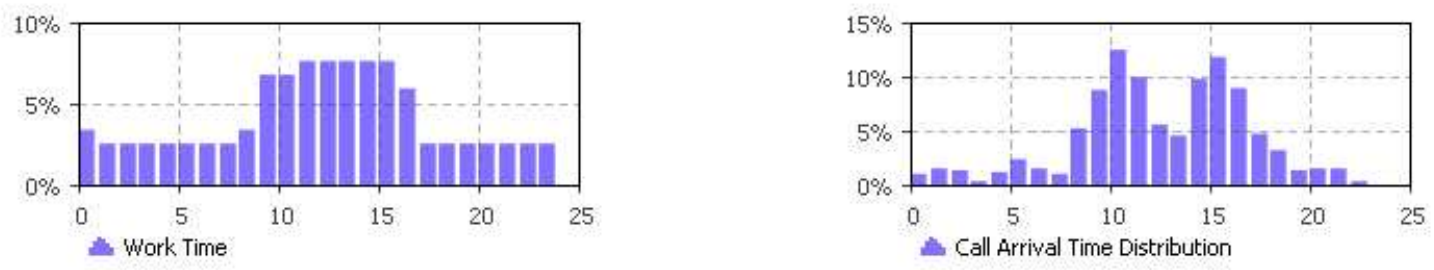

Figure 5: Distribution of work time and call arrivals [seconds] (ResourceAnalysts 2015).

In our simulation the service block fulfilled several roles: (1) limit the number of calls answered per hour by linking the calls to advisors through the resource pool; (2) logging queue times and advisors that waited less than 20 seconds; (3) allow calls to time out and leave the queue if their time to abandon was reached before interacting with an advisor; (4) set service time.

It was found from the real-world data that service times for different types of queues were very different. At this point MATLAB distribution fitting was used to calculate the distributions with best fit. Our data confirmed the results of a study by Brown et al. (2005) that lognormal rather than exponential or gamma were the best fit. Therefore the lognormal distribution was used for defining the base service times. The base service time was supplemented by the experience level of the current batch of staff.

As previously stated, it was not possible to implement true interaction between calls and agents. One way around this problem was to keep track of the total level of experience and apply this as a multiplier to the service time. This is not as effective as truly modelling a newly trained advisor with a break down call, but it is suitable to reflect the general effect that new starters have on a group of trained staff - they affect more than their own calls, as they have to ask questions of other workers and have a higher chance of needing to escalate calls to a manager. Table 4 presents the lognormal service time distributions for the different services as well as the definition of the service time modifier representing the experience level of the work crew. 


\section{May and Siebers}

Table 4: Lognormal service time distributions for the different services.

\begin{tabular}{|l|l|}
\hline Call Type & \multicolumn{1}{|c|}{ Service Time Distribution } \\
\hline Service & $(\operatorname{lognormal}(5.47917,0.506602,0) / 60) *$ vServiceTimeModifier \\
\hline Breakdown & $($ lognormal $(5.79643,0.614067,0) / 60) *$ vServiceTimeModifier \\
\hline vServiceTimeModifier= $(($ vStaffTraining*1.4)+(vStaffTrained*1.2)+(vStaffPermanent*1) $) /$ pNoCCAgentsAvailable \\
\hline
\end{tabular}

It was determined early in the process that there was a relationship between the weather and the number of incoming breakdown calls. Real-world data was used to compare the proportion of the customer base calling in with breakdowns to the mean temperature. Mean temperature data was used from the Met Office (Hadley Centre 2015) and is plotted against the call data in Figure 6.

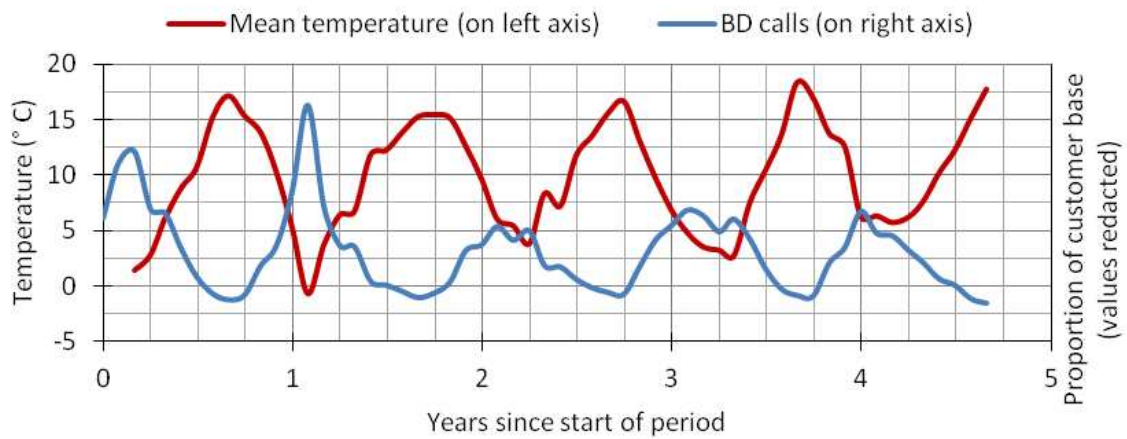

Figure 6: "breakdown calls as proportion of customers" compared to "mean temperature".

The correlation coefficient between breakdown calls as a proportion of customer base with degree-days was calculated using Excel, giving an R2 value of 0.88971. The correlation with mean temperature was found to be slightly lower, at -0.88120 . Once the relationship was known between calls and degree-days it could be expressed with Equation 1. A function was written to allow the user to choose between different severities of winter. Once a severity is chosen Equation 2 provides specific values for defining a Gaussian distribution for modelling the degree-day profile. Next the degree-day relationship equation is applied to calculate the breakdown call total for a month (Equation 3) and finally the service calls are added as $8 \%$ of the customer base to ensure every customer has a service (Equation 4). The result is shown in Figure 7.
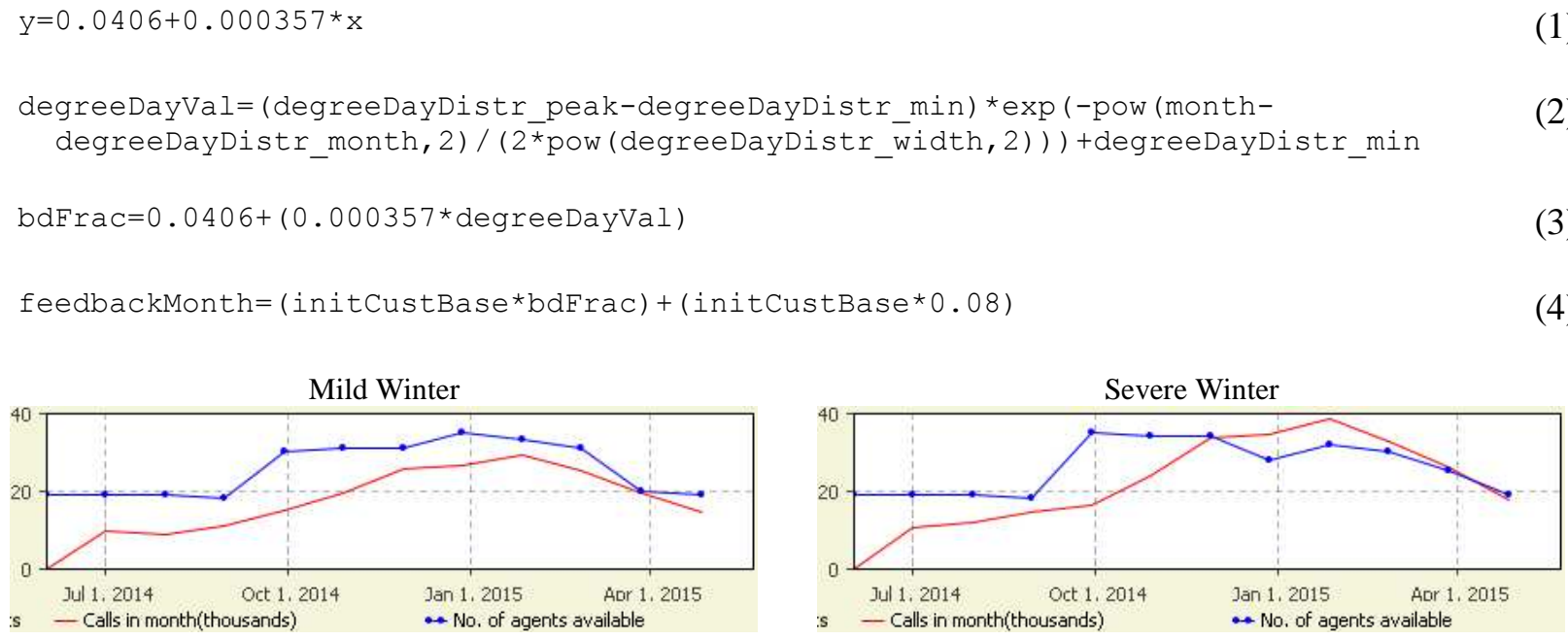

Figure 7: Mild and severe winter simulation calls by month. 
May and Siebers

\subsubsection{Graphical User Interface}

A lot of effort has gone into GUI design and usability testing as the tool is supposed to be used as a recruitment tool that supports managers (i.e. non computer scientists) in their decision making process. The tool was designed around Nielson's heuristic usability principles (Nielson 1993). An online version of the tool can be found at RunTheModel (2015). Having a look at the tool will make it easier to understand the following explanations.

- Input Screen: The user has four inputs to the simulation. The first two relate to staffing levels (August FTE staff; mid-October target FTE staff). The second two govern the incoming call rate (customer base; weather profile). The inputs are simple and also in the language of the user, e.g. FTE is used instead of number of advisors because of part-time staff.

- Output Screens: Once the model has been started, the application defaults to the Year Output screen, as it is the most useful for experienced users who just want to try a scenario and see the results. This screen contains a lot of text that is necessary for new users to understand the graphs. From this screen the user has a choice to look at the other output screens or jump to the Model Working screen. The additional output screens provide more detailed information about monthly and daily operation. It turned out that these screens were not as useful to the managers as the Year Output screen.

- Process Screen: This screen presents an overview of the two processes that are modelled (in form of flow charts) and provides real time information about all variables within the processes as well as all inputs, and outputs. The flow chart was added to help the user understand the simulation assumptions. In practice, this screen is of limited use to the CC manager end user as it is technical in nature, but it is useful as part of the system documentation, particularly for maintaining the code in the future.

\section{TESTING}

We have conducted conceptual model validation as well as face validation of the implemented model in collaboration with the different stakeholders. The valuable advice given by the employees was considered during our iterative model development. We have also done some usability testing of our prototype tool, which provided us with some very useful feedback regarding the usefulness of the different outputs. Recommended changes have been considered for the next iteration of the GUI development.

So far we have only conducted some light weight experiments to validate our prototype. One such experiment is about testing the relationship between "length of fixed term contract" and "weather severity". Three lengths of contracts were tested through the seasonal model and the outputs subjected to the Student t-distribution test of statistical significance. We did 10 replications for each scenario. The runtime for each replication was 1 year. The results of the experiment are presented in Table 5.

- Calls answered in less than 20 seconds: As expected severe weather always has a negative impact on targets and although it looks like the 3 month contract may be slightly better, no statistical significance found.

- Calls per agent per hour: 3 month contracts were found to be statistically significantly worse than both 6 and 9 month contracts during an average winter.

- Average time in system: 9 month contracts were found to be statistically better for service times than 3 month contacts, but no different than 6 month contracts during an average winter.

In conclusion, 9 month contracts give time for advisors to become better trained over the winter period and this may result in better service times for customers. However, 3 month contracts are more adaptable and allow managers to lose staff quickly if a winter turns out to be mild. A full cost benefit analysis was recommended as it is suggested that contract length has an effect on CC key performance indicators. Although the impact of the change in contract length is minimal, the proof of a relationship was used as evidence as part of a larger bid to improve recruitment practices. Further opportunities for experiments 
with the model include investigations of the impact of recruitment start date or different rota strategies, and optimization of training duration to balance new employee competence against time occupied with training.

Table 5: Results of the experiment.

\begin{tabular}{|l|r|r|r|}
\hline Calls answered less 20 seconds & Low weather severity & Avg. Weather severity & High weather severity \\
\hline 3 month contract & $93.1 \%$ & $90.8 \%$ & $89.0 \%$ \\
\hline 6 month contract & $92.0 \%$ & $89.7 \%$ & $88.0 \%$ \\
\hline 9 month contract & $93.3 \%$ & $89.7 \%$ & $88.2 \%$ \\
\hline Calls per agent per hour & Low weather severity & Avg. Weather severity & High weather severity \\
\hline 3 month contract & 4.3 & 4.6 & 4.8 \\
\hline 6 month contract & 4.4 & 4.7 & 4.9 \\
\hline 9 month contract & 4.3 & 4.7 & 4.9 \\
\hline Avg. time in system (minutes) & Low weather severity & Avg. Weather severity & High weather severity \\
\hline 3 month contract & 6.01 & 6.14 & 6.20 \\
\hline 6 month contract & 6.01 & 6.11 & 6.18 \\
\hline 9 month contract & 6.00 & 6.13 & 6.20 \\
\hline
\end{tabular}

\section{CONCLUSIONS}

In this paper we have presented the development of a recruitment tool that allows determining the best case scenario for timing hiring new staff and deciding the optimum length of temporary employment contracts. We have worked together with a boiler maintenance company on the conceptualization and testing of a prototype of such a tool. The usability tests conducted so far confirmed the usefulness of the tool for the management of the company to support their decision making processes. The prototype has now been handed over to the company for a long-term real-world test and we hope that we will be able to report on the outcome of this test and provide some results of experiments conducted during the test in our next WSC paper. One of the key limitations in our current model is that we are using passive agents, i.e. we do not take any form of bounded rational human behaviour into account. It is not clear yet what kind of impact that has on the accuracy of our prediction. Future work will therefore entail to design "suitable" intelligent agents for the job at hand (i.e. finding the right level of abstraction) and to test the impact on output accuracy, when using such agents. The foundations are already there as we can reuse the passive agents - we just need to put some life into them.

\section{REFERENCES}

Axelrod R. 1997. "Advancing the Art of Simulation in the Social Sciences." Complexity 3(2):16-22.

Bonabeau, E. 2002. "Agent-Based Modeling: Methods and Techniques for Simulating Human Systems." In Proceedings of the National Academy of Sciences 99(suppl 3):7280-7287.

British Gas. 2015. Contact Telephone Numbers. Accessed 9 July. http://www.britishgas.co.uk/help-andadvice/contactus-personal-details/contact-us.html.

Brown, L., N. Gans, A. Mandelbaum, A. Sakov, H. Shen, S. Zeltyn, and L. Zhao. 2005. "Statistical Analysis of a Telephone Call Center: A Queueing-Science Perspective" Journal of the American Statistical Association 100:36-50.

CelsiusPro. 2015. Weather Risk Management: A Challenge for the Energy Industry. Accessed July 9. http://www.celsiuspro.com/Portals/0/Downloads/Weather Risk Management for Utilities_White Paper.pdf.

CCA Global. 2015. Learning Zone FAQs. Accessed July 9. http://www.cca-global.com/gsx/content/ learningzone/?contentid=199. 
May and Siebers

CFA (BusinessSkills@Work). 2012. Contact Centre Operations Labour Market 2012. http://www.oph.fi/download/145729_Contact_Centres_LMI_2012_.pdf [Accessed July 9, 2015].

CWU Research. 2004. "Best Practice in Call Centres 2004". http:/www.cwu.org/uploads/documents/ callcentresbest.doc [Accessed July 9, 2015].

Forrester, J. W. 1997. "Industrial Dynamics." Journal of the Operational Research Society 48(10):10371041.

Koole, G. and A. Mandelbaum. 2002. "Queueing Models of Call Centers: An Introduction." Annals of Operations Research 113(1-4):41-59.

Ernst, A. T., H. Jiang, and M. Krishnamoorthy. 2004. "Staff Scheduling and Rostering: A Review of Applications, Methods and Models." European Journal of Operational Research 153(1):3-27.

Hadley Centre. 2015. HadCET Diagnostics and Data for the Current and Previous Years. Accessed July 9. http://www.metoffice.gov.uk/hadobs/hadcet.

Leombruni, R. and M. Richiardi. 2005. "Why are Economists Skeptical about Agent-Based Simulations?" Physica A: Statistical Mechanics and its Applications 355(1):103-109.

Meier, H. and K. Rehdanz. 2010. "Determinants of Residential Space Heating Expenditures in Great Britain." Energy Economics 32(5):949-959.

May, L. 2014. "Using Simulation to Assist Recruitment in Seasonally Dependant Contact Centres." MSc IT Dissertation, Nottingham University, Nottingham, UK.

Myron, D. 2002. "A Day in the Life of a Call Center". CRM Magazine Nov2002. http://www.destinationcrm.com/Articles/Editorial/Magazine-Features/A-Day-in-the-Life-of-a-CallCenter-47993.aspx [Accessed July 9, 2015].

Nielsen J. 1993. Usability Engineering. San Diego, CA: Academic Press.

nPower. 2015. Contact Us. Accessed July 9. https://www.npower.com/home/help-and-support/contactus/\#bills.

ResourceAnalysts. 2015. "Home » Workload Analysis » Call Centre". Accessed July 9. http://www.rostering.co.uk/workload-analysis-call-centre.

Robinson, S. 2014. Simulation: The Practice of Model Development, 2e. Chichester, UK: John Wiley.

SHRM. 2008. "Salaries as a Percentage of Operating Expense" http://www.shrm.org/research/ articles/articles/pages/metricofthemonthsalariesaspercentageofoperatingexpense.aspx [Accessed July 9, 2015].

RunTheModel. 2015. "BasicCallCentreV10". Accessed 9 July, 2015. http://www.runthemodel.com/ models/k-prkou5RLyMmz2Nv9gWiO.

Siebers, P. O. and U. Aickelin. 2008. "Introduction to Multi-Agent Simulation." In Encyclopaedia of Decision Making and Decision Support Technologies, edited by F. Adam, 554-564. Hershey, NY:IGI Global.

XJtek. 2015. "AnyLogic Simulation Software". Accessed July 9. http://www.anylogic.com.

\section{AUTHOR BIOGRAPHIES}

LEEANNE MAY is a postgraduate of the School of Computer Science, University of Nottingham, UK. She has an interest in the use of simulation and probabilistic models to analyze and inform commercial operations. Her email address is contact@leeannemay.net.

PEER-OLAF SIEBERS is a lecturer in the School of Computer Science, University of Nottingham, UK. He has an interest in object oriented Agent Based Modelling (ooABM) for simulating collective human behaviour. His email address is peer-olaf.siebers@nottingham.ac.uk. 